\title{
Functional Connectivity and Coactivation of the Nucleus Accumbens: A Combined Functional Connectivity and Structure-Based Meta-analysis
}

\author{
Franco Cauda $^{1,2}$, Andrea E. Cavanna ${ }^{3,4}$, Federico D'agata ${ }^{1,2,5}$, \\ Katiuscia Sacco $^{1,2}$, Sergio Duca ${ }^{1}$, and Giuliano C. Geminiani ${ }^{1,2}$
}

\begin{abstract}
This article investigates the functional connectivity patterns of the nucleus accumbens (NAcc) in 18 healthy participants using a resting state functional connectivity (rsFC) protocol. Also, a meta-analytic connectivity modeling (MACM) was used to characterize patterns of functional coactivations involving NAcc: The results of a structure-based meta-analyses of 57 fMRI and PET studies were submitted to activation likelihood estimation analysis to estimate consistent activation patterns across the different imaging studies. The results of the combined rsFC and MACM analyses show that spontaneous activity in NAcc predicts activity in regions implicated in reward circuitries, including orbitomedial prefrontal cortex, globus pallidus, thalamus, midbrain, amygdala, and insula. This confirms the key role of NAcc in the mesocorticolimbic system, which integrates inputs from limbic and cortical
\end{abstract}

\section{INTRODUCTION}

The nucleus accumbens (NAcc) is increasingly recognized as a pivotal center within brain systems regulating motivation and reward. NAcc is located at the conjunction between the head of the caudate and the anterior portion of the putamen, laterally to the septum pellucidum (Groenewegen, Wright, Beijer, \& Voorn, 1999; Groenewegen, Wright, \& Beijer, 1996; Heimer, Zahm, \& Alheid, 1995); together with the olfactory tubercle, NAcc forms the ventral striatum, a critical element of the mesocorticolimbic system (Heimer \& Wilson, 1995).

Although NAcc is traditionally described as a collection of neuronal ensembles with different functional and behavioral connotations within the BG (Pennartz, Groenewegen, \& Lopes da Silva, 1994), it is widely accepted that there are two major functional components, the core and the shell, which are characterized by specific input and output channels (Surmeier, Ding, Day, Wang, \& Shen, 2007).

Some of the main afferent projections to NAcc are from the cortex, more specifically from the orbitomedial

\footnotetext{
${ }^{1}$ Koelliker Hospital, Turin, Italy, ${ }^{2}$ University of Turin, ${ }^{3}$ University College London, ${ }^{4}$ University of Birmingham and BSMHFT, ${ }^{5}$ AOU San Giovanni Battista, Turin, Italy
}

regions. We also detected activity in brain regions having few or no direct anatomical connections with NAcc, such as sensorimotor cortex, cerebellum, medial and posterior parietal cortex, and medial/inferior temporal cortex, supporting the view that not all functional connections can be explained by anatomical connections but can also result from connections mediated by third areas. Our rsFC findings are in line with the results of the structure-based meta-analysis: MACM maps are superimposable with NAcc rsFC results, and the reward paradigm class is the one that most frequently generates activation in NAcc. Our results overlap considerably with recently proposed schemata of the main neuron systems in the limbic forebrain and in the anterior part of the limbic midbrain in rodents and nonhuman primates.

prefrontal cortex (OMPFC), that is, Brodmann's areas 11, 13, 24, 25, and 32 (Haber \& McFarland, 1999). OMPFC inputs to NAcc are massive and represent the defining feature of separate functional circuits (Goldman-Rakic \& Selemon, 1986) involving OFC, ACC (Parkinson, Willoughby, Robbins, \& Everitt, 2000), and medial prefrontal cortex (MPFC) (Carmichael \& Price, 1994). It has been proposed that OMPFC-NAcc pathways play a key role in the development of reward-guided behaviors by linking reward experiences with their motivational and emotional features (Cummings, 1995).

NAcc is also interconnected with several subcortical structures, namely hippocampal region, midline, medial parafascicular, medial dorsal (MDN) and intralaminar thalamic nuclei, ventral pallidum, dopaminergic ventral tegmental and retrorubral cell groups, basal amygdaloid complex, dorsal and medial raphe nucleus, and noradrenergic cell group in the nucleus of the solitary tract (Morgane, Galler, \& Mokler, 2005; Groenewegen et al., 1996, 1999; Brog, Salyapongse, Deutch, \& Zahm, 1993). Of note, the projections of these structures are not restricted to NAcc but extend to the olfactory tubercle, caudate, putamen, and more caudal ventral striatal areas. Therefore, NAcc integrates the limbic and cortical inputs and projects to other BG nuclei, which send feedback projections into the prefrontal cortex 
via the MDN (Zahm, 1998; O’Donnell, Lavin, Enquist, Grace, \& Card, 1997).

The connectivity pattern of NAcc provides support to the traditional hypothesis that NAcc constitutes the neural substrate for limbic-motor interactions (Mogenson, Jones, \& Yim, 1980). More recently, neurochemical studies have suggested that NAcc plays a prominent role in the reward and motivation process via dopaminergic innervation (Knutson \& Cooper, 2005; Glimcher \& Rustichini, 2004; McClure, York, \& Montague, 2004; Schultz, 2004). Current research on the behavioral correlates of NAcc focuses on the role of the mesolimbic dopamine system in reward and reinforcement (Wise, Bauco, Carlezon, \& Trojniar, 1992) and, to a lesser extent, in response selection and intensification processes (Redgrave, Prescott, \& Gurney, 1999; Salamone, Cousins, \& Snyder, 1997; Robbins \& Everitt, 1992) or arousing and preparatory effects of reinforcers (Robbins \& Everitt, 1992; Taylor \& Robbins, 1984; Koob, Riley, Smith, \& Robbins, 1978).

NAcc has been found to be recruited in multiple forms of positive (Burgdorf \& Panksepp, 2006) and negative (Carretie, Albert, Lopez-Martin, \& Tapia, 2009) affective states; likewise, functional neuroimaging studies have found activity in the human accumbens following both rewarding (Mobbs, Greicius, Abdel-Azim, Menon, \& Reiss, 2003; Aharon et al., 2001) and aversive stimuli (Gottfried, O’Doherty, \& Dolan, 2002; Becerra, Breiter, Wise, Gonzalez, \& Borsook, 2001). Moreover, several differential functions within each component of NAcc have been reported in animal studies (McFarland, Lapish, \& Kalivas, 2003; Grill \& Coghill, 2002; Zahm, 1999), albeit not in humans. Finally, disturbances at the level of NAcc have been implicated in drug abuse and schizophrenia, in addition to affective disorders (Kienast \& Heinz, 2006; Totterdell, 2006).

Despite clear-cut clinical relevance, our knowledge of NAcc connectivity is mainly based on experimental studies using tractographic techniques in animals (Haber, Kim, Mailly, \& Calzavara, 2006; Middleton \& Strick, 1994, 2002; Ferry, Ongur, An, \& Price, 2000; Haber, Fudge, \& McFarland, 2000; Cavada \& Goldman-Rakic, 1991; Selemon \& GoldmanRakic, 1985). The literature in humans has flourished only recently, with 2 diffusion tensor imaging (DTI) studies (Leh, Ptito, Chakravarty, \& Strafella, 2007; Lehericy et al., 2004), one on fMRI resting state functional connectivity (rsFC) (Di Martino et al., 2008) and one on meta-analysis of coactivation patterns, from $126 \mathrm{fMRI}$ and PET studies (Postuma \& Dagher, 2006). However, the primary goal of these studies was the investigation of BG functional subdivisions and connectivity patterns; none of them was specifically targeted at NAcc. Thus, we set out to investigate the connectivity pattern of NAcc in 18 healthy volunteers by using an rsFC protocol and comparing our findings with a structure-based meta-analysis (Laird, Eickhoff, Kurth, et al., 2009) of 59 fMRI and PET studies.

rsFC (Fox \& Raichle, 2007; Margulies et al., 2007; Vincent et al., 2007; Damoiseaux et al., 2006; Fox et al., 2005; Greicius, Krasnow, Reiss, \& Menon, 2003; Hampson,
Peterson, Skudlarski, Gatenby, \& Gore, 2002) is a recently developed technique that allows in vivo assessment of brain networks by detecting coherent patterns of spontaneous activity in the resting brain. It has been shown that correlations in slowly fluctuating spontaneous brain activity tend to reflect intrinsic functional networks. Resting state networks (RSNs) are localized in the gray matter and are likely related to ongoing neuronal activity, as demonstrated by aliasing of cardiac and respiratory cycles (De Luca, Beckmann, De Stefano, Matthews, \& Smith, 2006). Moreover, RSNs are characterized by changes in BOLD signals that are comparable with task-related changes (i.e., up to 3\%) and are consistent across individuals and stable across repeated measurements (Damoiseaux et al., 2006).

Structure-based meta-analyses focus on specific anatomical regions and address global coactivation patterns across a diverse range of tasks and experimental designs. The consistency of coactivation patterns across experiments is interpreted as a piece of evidence for the functional connection of groups of regions; this type of functional connectivity has also been indicated as meta-analytic connectivity modeling (MACM) (Robinson, Laird, Glahn, Lovallo, \& Fox, 2009). In fact, two recent meta-analysis studies (Smith et al., 2009; Toro, Fox, \& Paus, 2008) demonstrated that the set of major covarying activation networks identified from largescale meta-analyses overlaps almost completely with the set of networks that are visualized in the resting brain. These results provide strong evidence that RSNs reflect functional neural networks and that these dynamic networks are engaged even at rest (Fox \& Raichle, 2007). Therefore, similar to previous studies (Toro et al., 2008; Postuma \& Dagher, 2006; Lancaster, Laird, Fox, Glahn, \& Fox, 2005; Koski \& Paus, 2000), we used MACM (Laird, Eickhoff, Kurth, et al., 2009) to characterize patterns of functional coactivations in the human brain and compared the emerging patterns with the results of our rsFC analysis to validate each other.

\section{METHODS}

\section{Subjects}

Eighteen right-handed healthy volunteers (nine women; mean age $=51.2$ years, $S D=19.2$ years), free of neurological or psychiatric disorders, not taking medications known to alter brain activity, and with no history of drug or alcohol abuse, participated in the study. Written informed consent was obtained from each subject, in accordance with the Declaration of Helsinki; the study was approved by our institutional committee on ethical use of human subjects. The fMRI study was performed at the Ospedale Koelliker in Turin.

\section{Task and Image Acquisition}

Subjects were instructed to simply keep their eyes closed, to think of nothing in particular, and not to fall asleep. 
After the scanning session, participants were asked if they had fallen asleep during the scan, and data from subjects with positive or doubtful answers were excluded from the study.

Images were gathered on a 1.5-Tesla INTERA scanner (Philips Medical Systems, Andover, MA) with a SENSE highfield, high-resolution (MRIDC) head coil optimized for functional imaging. Resting state functional T2*-weighted images were acquired using EPI sequences, with a repetition time of $2000 \mathrm{msec}$, an echo time of $50 \mathrm{msec}$, and a $90^{\circ}$ flip angle. The acquisition matrix was $64 \times 64$, with a 200-mm field of view. A total of 200 volumes were acquired, with each volume consisting of 19 axial slices parallel to the anteriorposterior (AC-PC) commissure; slice thickness was $4.5 \mathrm{~mm}$ with a $0.5-\mathrm{mm}$ gap. To reach a steady-state magnetization before acquiring the experimental data, two scans were added at the beginning of functional scanning: The data from these scans were discarded.

Within a single session for each participant, a set of three-dimensional high-resolution $\mathrm{T}_{1}$-weighted structural images was acquired, using a fast field echo sequence, with a repetition time of $25 \mathrm{msec}$, an ultrashort echo time, and a $30^{\circ}$ flip angle. The acquisition matrix was $256 \times 256$; the field of view was $256 \mathrm{~mm}$. The set consisted of 160 contiguous sagittal images covering the whole brain. In-plane resolution was $1 \times 1 \mathrm{~mm}$, and slice thickness was $1 \mathrm{~mm}$ $\left(1 \times 1 \times 1 \mathrm{~mm}^{3}\right.$ voxels $)$.

\section{Selection of NAcc ROIs}

For rsFC analysis, two bilateral anatomical ROIs were drawn according to the AFNI brain structure atlas (afni.nimh.nih. gov/afni/doc/misc/afni ttatlas/). The mean ROI volume was $143 \mathrm{~mm}^{3}$ (see Figure $\overline{1}$ ).
For MACM analysis, we used rectangular ROIs, because BrainMap does not currently allow to draw anatomical ROIs. Therefore, two rectangular ROIs were drawn bilaterally according to the Talairach Daemon Database (Lancaster et al., 2000) around the following coordinates: left, $X=-10 Y=8$ $Z=-8$; right, $X=12 Y=7 Z=-8$ (see Figures 1 and 2 ). ROIs for activation likelihood estimation (ALE) metaanalysis were bigger than the rsFC ROIs to account for the nonanatomical ROI shape and the mean "betweentemplate variance" introduced by different normalization strategies between different studies (Eickhoff et al., 2009). The boundaries for the right ROI were $(8,4,-12)$ to $(16,14$, $-5)$; the boundaries for the left ROI were $(-15,5,-12)$ to $(-7,13,-5)$.

\section{DATA ANALYSIS}

BOLD imaging data were analyzed using BrainVoyager QX software (Brain Innovation, Maastricht, Holland); an inhouse developed Matlab Script has been used to create masks (see Supplementary Data for a description). Functional images were preprocessed as follows to reduce artifacts (Miezin, Maccotta, Ollinger, Petersen, \& Buckner, 2000): (i) slice scan time correction was performed using a sinc interpolation algorithm; (ii) 3-D motion correction was applied: using a trilinear interpolation algorithm, all volumes were spatially aligned to the first volume by rigid body transformations and the rototranslation information was saved for subsequent elaborations; (iii) spatial smoothing was performed using a Gaussian kernel of $8 \mathrm{~mm}$ FWHM; (iv) temporal filtering (linear trend removals) and a band pass filter of 0.01-0.08 Hz was used (as described in Greicius et al., 2003; Biswal, Yetkin, Haughton, \& Hyde, 1995), as it has been shown that the $0.01-$ to $0.08-\mathrm{Hz}$ frequency range
Figure 1. Spatial distribution of the ROI used as seed regions for rsFC analyses. Anatomical ROIs for functional connectivity were drawn according with the AFNI data collection. ROIs for ALE meta-analysis were drawn using the subsequent limits: left NAcc, $-15,5,-12$ to $-7,13$, -5 ; right NAcc, $8,4,-12$ to 16 , $14,-5$.

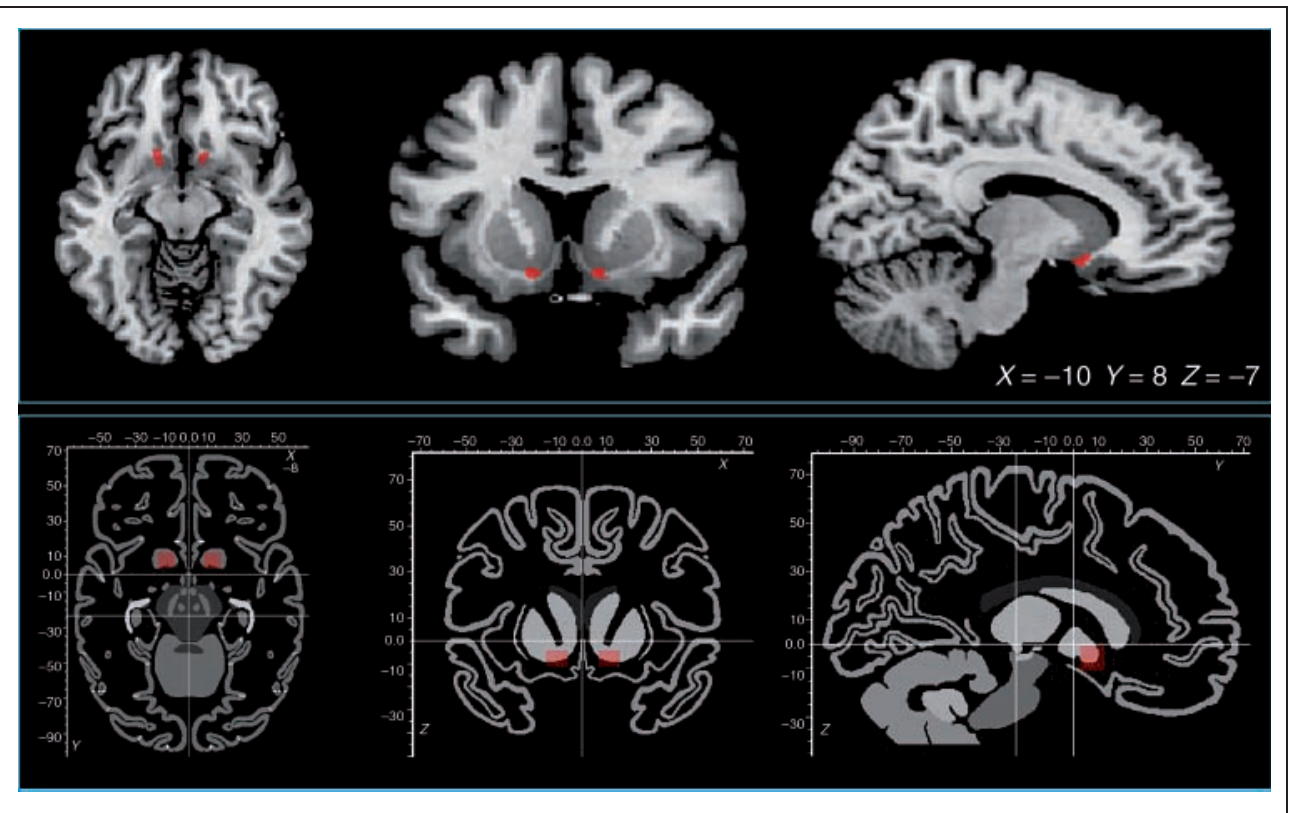


Figure 2. Spatial distribution of the foci resulted from the structure-based meta-analysis.

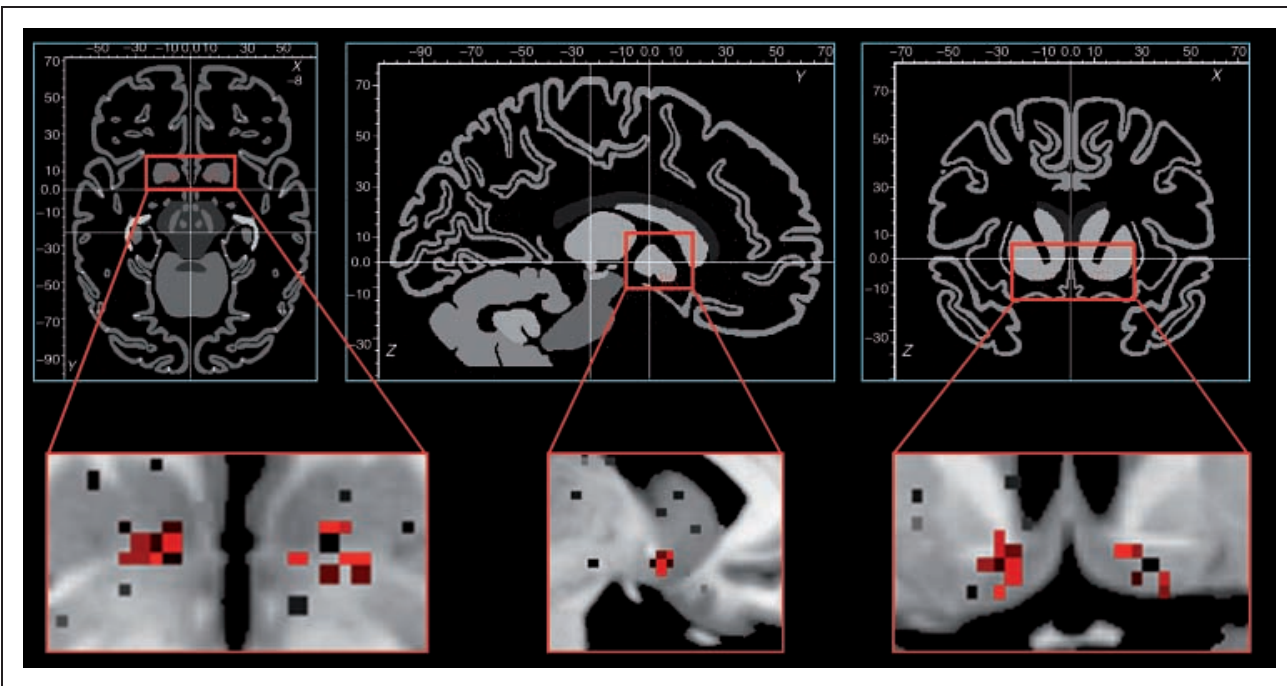

had the greatest power in revealing the underlying connectivity (Greicius, Supekar, Menon, \& Dougherty, 2009; Hagmann et al., 2008; Vincent et al., 2007; Achard, Salvador, Whitcher, Suckling, \& Bullmore, 2006; Fransson, 2006; Biswal et al., 1995).

Preprocessing was followed by a series of steps to facilitate accurate anatomical localization of brain activity and intersubject averaging. For each subject, the slice-based functional scan was coregistered with the 3-D high-resolution structural scan, and the 4-D data set was transformed into Talairach space (Talairach \& Tournoux, 1988).

The first step to perform all FC analyses was to extract BOLD time courses from each ROI (defined as described above) by averaging over voxels within each region. Several nuisance covariates were included in our analyses to control for the effects of physiological processes (such as fluctuations related to cardiac and respiratory cycles) (Bandettini \& Bullmore, 2008; Birn, Murphy, \& Bandettini, 2008; Napadow et al., 2008) and motion. Specifically, we included nine additional covariates that modeled nuisance signals from white matter, global signal (Fox, Zhang, Snyder, \& Raichle, 2009), and CSF, as well as six motion parameters.

All seed-based predictors were $z$-normalized; temporal autocorrelation correction (Woolrich, Ripley, Brady, \& Smith, 2001) was used. Seed ROI-driven FC maps were computed on a voxel-wise basis for each previously selected region. The individual participant multiple regression analysis was carried out using the general linear model (Friston, Ashburner, Kiebel, Nichols, \& Penny, 2007) and resulted in a $t$-based map (SPMt) FDR corrected for multiple comparisons (Genovese, Lazar, \& Nichols, 2002; Benjamini \& Hochberg, 1995) $(q<0.05$, cluster threshold $K>10$ voxels in the native resolution).

\section{Group Statistical Map}

Random effect group level analyses, controlling for age and gender effects, were conducted using the BrainVoyager QX ANCOVA module $[p<.05$ FDR corrected (Genovese et al.,
2002; Benjamini \& Hochberg, 1995), cluster threshold $K>10$ voxels in the native resolution].

To evaluate the spatial consistency of functional connectivity patterns across subjects, we computed spatial probabilistic maps. The probability map describes the relative frequency (expressed in percentage) wherein the same network is represented over different brain areas.

To achieve maximum precision in the location description, we developed a Matlab script (see Supplementary Data) that, in case of large blobs, splits the activation in different BAs and Gyri that compose the original blob. When the script finds a voxel that does not match the BA mask or the Gyral mask (e.g., white matter voxels), this voxel is labeled as "Out of Gyrus."

\section{Reliability Test}

To evaluate the spatial consistency of functional connectivity patterns across subjects, we computed the split half reliability index: We calculated the reliability coefficient with the Spearman-Brown (Brown, 1910; Spearman, 1910) formula, $r_{\mathrm{sb}}=\frac{2 r_{\mathrm{h}}}{1+r_{\mathrm{h}}}$, wherein the term $r_{\mathrm{h}}$, in our case, is the spatial similarity of the maps obtained by two random selected, equally numerous subgroups. The $r_{\mathrm{h}}$ term is a measure of the intersection of two fuzzy sets, the Sørensen index (Sorensen, 1948), defined as: $Q S=\frac{2 C}{A+B}$, wherein $A$ and $B$ are the elements in sample $\mathrm{A}$ and $\mathrm{B}$, respectively, and $C$ is the number of elements shared by the two samples (this is equivalent to Dice metric).

\section{Structure-Based Meta-analysis}

We followed the workflow indicated by Laird, Eickhoff, Kurth, et al. (2009) for the structure-based meta-analyses, also indicated as MACM (Laird, Eickhoff, Li, et al., 2009; Robinson et al., 2009): (i) we extracted from the BrainMap database (Laird, Lancaster, \& Fox, 2005) all the studies involving only normal subjects that reported an activation in 
at least one of the two NAcc ROIs previously described, irrespective of class or behavioral paradigm that had generated that activation. The specific query was [Diagnosis = Normals] AND [ROIs = "left accumben", $(-15,5,-12)$ to $(-7,13,-5)$ OR "right accumbens", $(8,4,-12)$ to $(16,14$, $-5)$ ] and yielded a total of 42 articles (see Supplementary Table 1) corresponding to 57 experiments, leading to a total of 762 foci (see Figure 2).

BrainMap (Laird et al., 2005) is a database of published functional neuroimaging studies (mainly PET and fMRI) that contains both metadata descriptions of experimental design and activation locations in the form of stereotactic coordinates. BrainMap contains 1843 neuroimaging publications that describe analyses from 8618 experimental contrasts using 81 unique paradigm classes, yielding 69,681 locations (November 22, 2009).

\section{Activation Likelihood Estimation}

An ALE meta-analysis (Laird et al., 2005; Turkeltaub, Eden, Jones, \& Zeffiro, 2002) was performed on the sets of coordinates identified as coactivated during each ROI activation. Regions of convergence were calculated using GingerAle 2.0.

The ALE analysis is a quantitative method that can be used to estimate consistent activation across different imaging studies (Laird, Eickhoff, Kurth, et al., 2009). ALE maps of coactivations are derived based on patterns of foci of interest, where multiple studies have reported statistically significant peak activation. To limit the intersubject and interlaboratory variability, we used an algorithm that estimates the spatial uncertainty of each focus, taking into account the possible differences among the neuroimaging studies (Eickhoff et al., 2009). This algorithm was preferred to a prespecified FWHM as in the original ALE approach. The advantage of such an algorithm is that it limits the meta-analysis to an anatomically constrained space specified by a gray matter task. Furthermore, it comprises a method to calculate the above-chance clustering between experiments (i.e., random effects analysis) rather than between foci (fixed effects analysis; Eickhoff et al., 2009).

The original studies contributing these foci for each domain are presented in Supplementary Table 5.

\section{Paradigm Class Profiles}

Aside from MACM, we were interested in examining what mental processes are underpinned by the activation of our ROI. In BrainMap, metadata are organized under three experiment level fields: context, paradigm class, and behavioral domain. The "context" represents the purpose for which an experiment was designed. Possible contexts include normal mapping, age effects, disease effects, etc. The "paradigm class" is the experimental task isolated by the contrast. For a given experiment, multiple paradigm classes may apply. Paradigm classes include, among others, action observation, episodic recall, task switching, etc. A complete list of BrainMap's paradigm domains can be accessed at brainmap.org/scribe/.

\section{RESULTS}

Both rsFC and MACM techniques revealed a pool of areas that were connected to NAcc: these included putamen, caudate head, anterior and posterior cingulate, subcallosal gyrus, thalamus (MDN nucleus), medial frontal gyrus, amygdala, inferior parietal lobule, insula, caudate body, globus pallidus, parahippocampal gyrus, inferior frontal gyrus, culmen, precuneus, and cerebellum (Figures 3-6).

In addition, the rsFC analysis showed functional connections with the middle temporal gyrus, superior frontal gyrus, superior temporal gyrus, middle frontal gyrus, inferior temporal gyrus, supramarginal gyrus, fusiform gyrus, hippocampus, precentral gyrus, angular gyrus, hypotalamus, and pulvinar. Finally, MACM identified connections with the postcentral gyrus and the inferior occipital gyrus, which were missed by the rsFC technique (Figures 3-6).

Overall, the two techniques generally converge, but rsFC seems to have a better sensibility showing a richer pool of connected areas. It has to be considered that these two connectivity techniques are based on very different types of data: rsFC uses resting state scans, whereas MACM uses activation paradigms (see Figures 3-5, Supplementary Figures 1 and 2, and Supplementary Tables 1 and 2). A summary image can be seen in Supplementary Figure 8.

\section{Paradigm Class Profiles}

The paradigm class profiles, which more frequently lead to an activation in NAcc, are the reward tasks. Error detection, lexical decisions, and rest were all associated with weaker activations (see Supplementary Figure 6).

\section{Lateralization}

We repeated the FC analysis by using monolateral ROIs and by comparing the maps generated by right ROIs with the maps generated by left ROIs for both rsFC and MACM results (two-sample $t$ test, $p<.05$ FDR corrected, $K>10$ voxels in the native resolution). Lateralization analysis of the rsFC findings evidenced that the right NAcc is more connected with uncus, subcallosal gyri, insulae, parahippocampal gyri, and cerebellum whereas the left NAcc is more connected with OMPFC, subgenual, temporal, medial prefrontal, and posteromedial cortices (Figures 7 and 8, Supplementary Figures 3 and 4, and Supplementary Tables 3 and 4). Lateralization analysis of the MACM findings showed a similar pattern of lateralization: The insular, thalamic, anterior cingulated, pontine, and cerebellar areas are more right-lateralized, whereas the subgenual, motor/premotor, prefrontal, and occipital cortices are more left-lateralized (Figures 7 and 8, Supplementary Figures 3 and 4, and Supplementary Tables 3 and 4). 
Figure 3. NAcc resting state connectivity analysis. One sample $t$ test, FDR corrected: $q<0.05$, cluster threshold $K>10$ voxels in the native resolution. Maps projected on a mixed 2-D/3-D template with Brainvoyager QX 2.0.

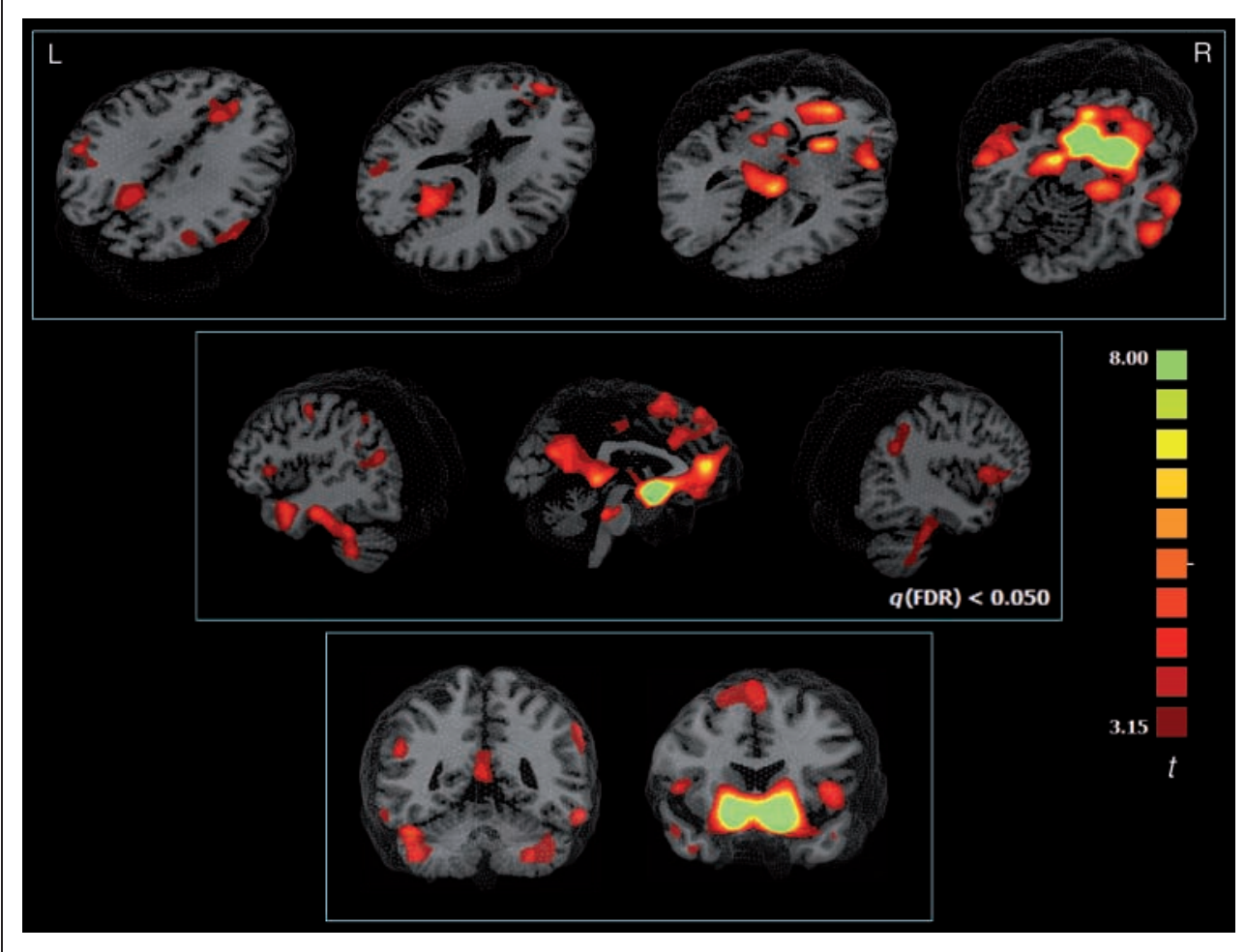

We assessed possible differences in the paradigm class profiles activating the left and right NAcc: The right NAcc is more activated by emotional paradigms, whereas the left NAcc is more activated by pain paradigms (see Supplementary Figure 7).

\section{Reliability Indexes}

The split-half test performed with the Spearman-Brown method between each ROI in the two split groups shows our results: $r_{\mathrm{sb}}=0.73$.
Figure 4. NACC MACM

connectivity analysis.

Results from the activation

likelihood estimation $(q<0.05$, $K>100 \mathrm{~mm}^{3}$ ). ALE maps generated with GingerAle 2.0 Maps projected on a mixed 2-D/3-D template with Brainvoyager QX 2.0 .

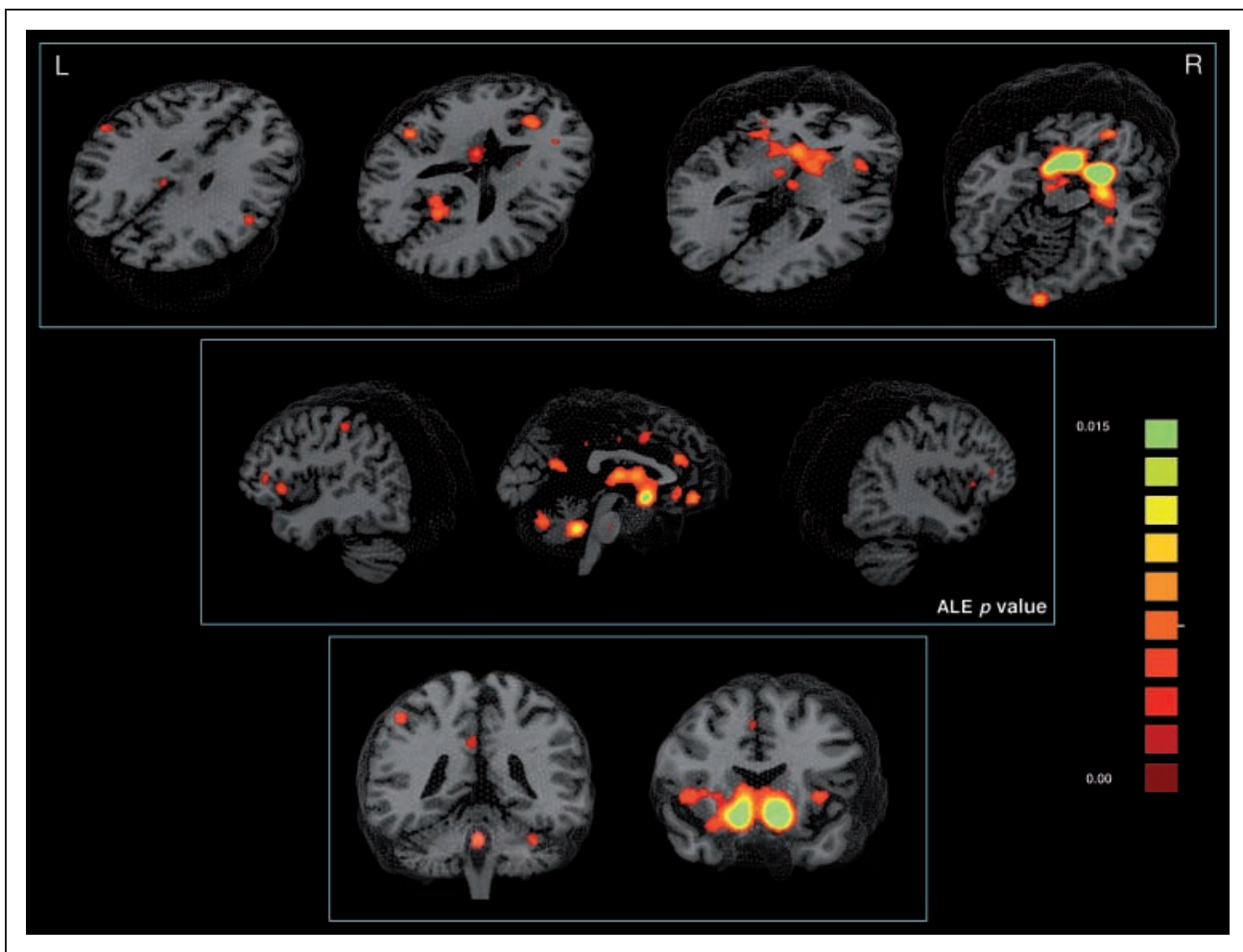


Figure 5. Quantitative differences between MACM and rsFC. The graph shows the quantitative difference in connectivity between rsFC and MACM in the regions where strongest connectivity was found. Red line = MACM; blue line $=$ rsFC.
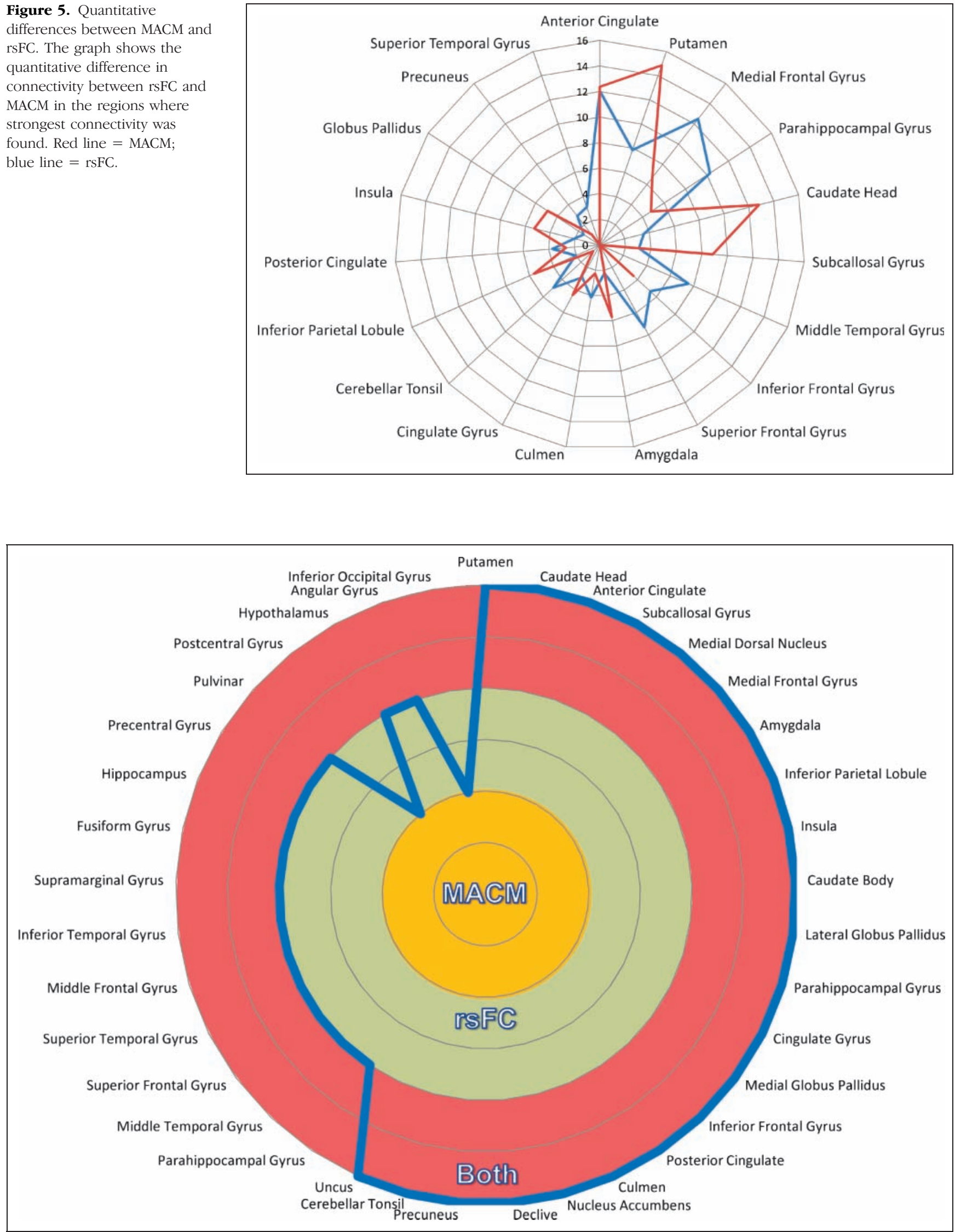

Figure 6. Qualitative differences between MACM and rsFC. The graph shows the areas where both rsFC and MACM show connections plus the areas characterized by significant connections according to rsFC only or MACM only. Yellow area = only MACM; green area = only rsFC; red area $=$ MACM and rsFC. 
Probability maps computed for assessing the spatial consistency and reproducibility of seed-generated maps yielded a high level of overlap among specific ROI-related rsFC maps for each subject (Figure 8 and Supplementary Figure S4).

\section{DISCUSSION}

To the best of our knowledge, this is the first study combining rsFC with MACM to investigate NAcc connectivity. We evaluated the functional network associated with NAcc activity by examining temporally correlated patterns of low-frequency spontaneous activity during rest in a group of 18 right-handed healthy volunteers and MACM derived from the representative sample of the fMRI and PET literature present in the BrainMap database. Our findings are consistent with the results of both animal models (Postuma \& Dagher, 2006; Morgane et al., 2005; Haber \& McFarland, 1999) and DTI and fMRI investigations in humans (Stoeckel et al., 2009; Di Martino et al., 2008; Postuma \& Dagher, 2006; Knutson \& Cooper, 2005; Becerra et al., 2001; Breiter \& Rosen, 1999). Spontaneous activity in NAcc predicted activity in regions implicated in reward circuitries, including OMPFC, globus pallidus, thalamus, midbrain, amygdala, and insula. Furthermore, we detected activity in brain regions that are described to have few or no direct connection with NAcc, such as sensorimotor cortex, cerebellum, medial and posterior parietal cortex, and medial/ inferior temporal cortex (Haber \& McFarland, 1999). It has been shown that functional connectivity patterns result not only from direct connections but also from connections mediated by third areas, thus suggesting that not all functional connections can be explained by anatomical connections, although FC overlaps considerably with tract tracing analysis (Damoiseaux \& Greicius, 2009). Consequently, our FC patterns give a representation of the brain regions working together with NAcc as a coordinate network and can be, in great part, explained by indirect connections (Damoiseaux \& Greicius, 2009). For example, several areas directly connected with NAcc, such as insula (Cauda et al., 2011), dorsal striatum, and thalamus, are characterized by strong connections with sensorimotor as well as parietal and cerebellar cortices. These observations are further validated by our MACM results, which essentially replicate the rsFC findings with the exception of the wider connectivity pattern with the fronto-temporal neocortex.

NAcc is a key element of the mesocorticolimbic system, which integrates inputs from limbic and cortical regions, linking motivation with action (Mogenson et al., 1980). Specifically, NAcc has a well-established role in mediating
Figure 7. NAcc resting state connectivity lateralization. Twosample $t$ test, FDR corrected: $q<0.05$, cluster threshold $K>10$ voxels in the native resolution. Colors from red to yellow indicate a prevalent right lateralization. Colors from blue to green indicate a prevalent left lateralization. Maps projected on a 2-D template with Brainvoyager QX 2.0.

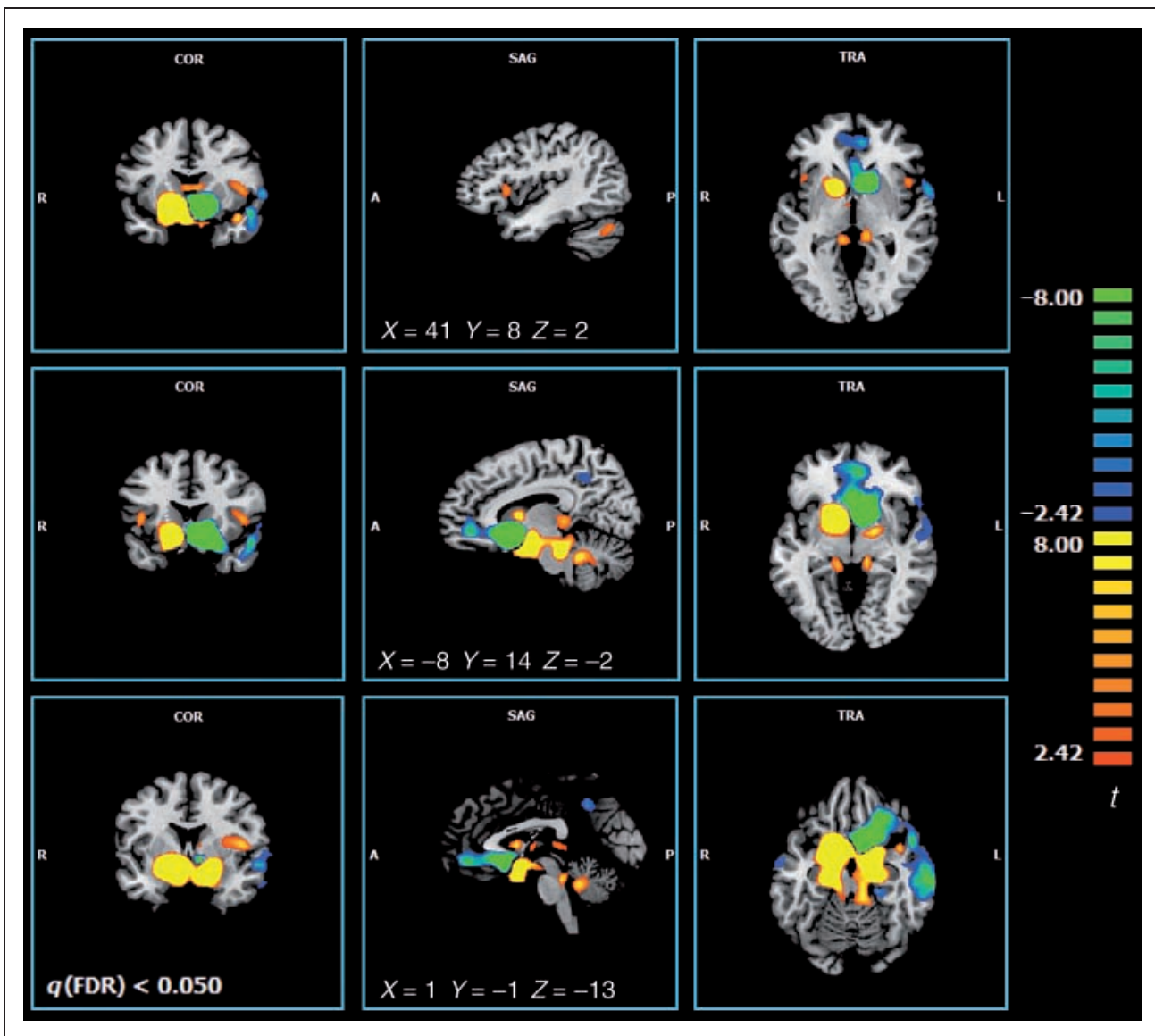


Figure 8. NACC MACM connectivity lateralization. Colors from red to yellow indicate a prevalent right lateralization. Colors from blue to green indicate a prevalent left lateralization. Maps projected on a 2-D template with Brainvoyager QX 2.0.

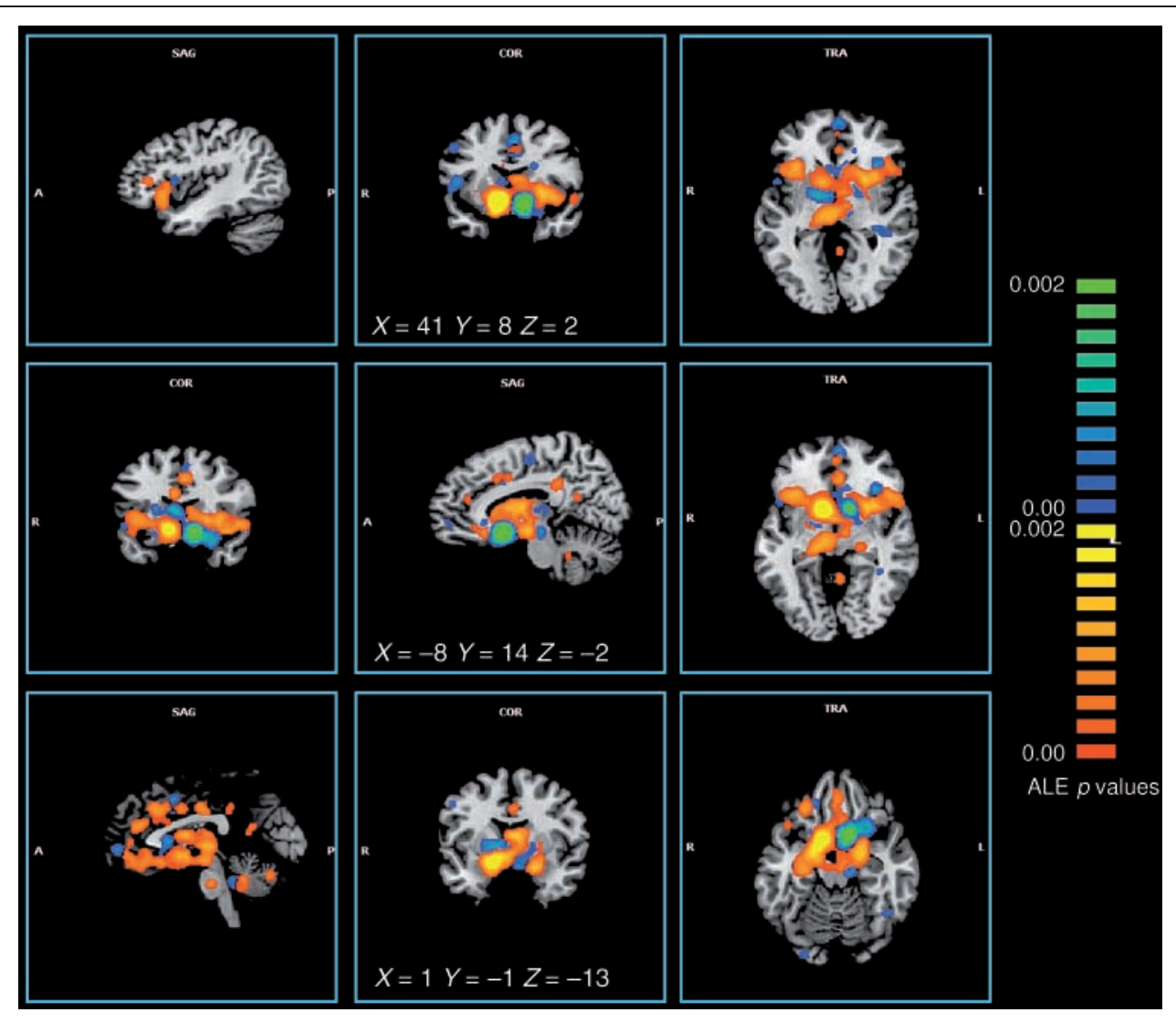

the rewarding effects of drug abuse and fundamental rewards such as food and sexual behavior (Carlezon \& Thomas, 2009). Interestingly, our results overlap considerably with recently proposed schemata of the principal neuron systems in the limbic forebrain and in the anterior part of the limbic midbrain in rodents and nonhuman primates (Figure 4) (Morgane et al., 2005; de Olmos \& Heimer, 1999; Haber \& McFarland, 1999). We found that NAcc positively correlated with OMPFC, insulae, MDN, amygdalae, and hippocampi. Of these, OMPFC, insulae, and MDN are known to be active during reward (Breiter \& Rosen, 1999), whereas the strong interconnections between NAcc, amygdala (Cardinal, Parkinson, Hall, \& Everitt, 2002), and hippocampus (O’Donnell \& Grace, 1995) are well described in the modified model of Lawrence, Sahakianb, and Robbins (1998) on corticostriatal circuits.

Our rsFC findings are further confirmed by the results of the structure-based meta-analysis: the reward paradigm class most frequently generates activation in NAcc. The ALE maps of the meta-analytical connectivity modeling are similar to the rsFC maps (Supplementary Table 5), thus confirming the validity of the rsFC results. The fact that in the midbrain we only found substantia nigra to be connected with NAcc can be explained by taking into account the relative low resolution of our method: Relatively small structures like ventral tegmental or dorsal raphe nucleus can be too small to be detected. Likewise, the small mid- line thalamic nuclei that are described as interconnected with NAcc (Haber \& McFarland, 1999) might be under the threshold of our resolution power; in fact, we detected only a significant cluster in the MDN nucleus, one of the largest thalamic nuclei known to have strong connections with several regions in the prefrontal and limbic regions (Cauda et al., 2009; Zhang et al., 2008; Morgane et al., 2005; McFarland \& Haber, 2002), including pallido-thalamic fibers belonging to the ventral striatal or limbic loop (Alexander, DeLong, \& Strick, 1986; Haber, Groenewegen, Grove, \& Nauta, 1985).

Our results are in agreement with most DTI, fMRI, and meta-analytic (Di Martino et al., 2008; Postuma \& Dagher, 2006; Breiter \& Rosen, 1999) studies in the literature. There are, however, a few differences. For example, a meta-analysis of 126 PET and fMRI studies by Postuma and Dagher (2006) failed to demonstrate connections with OFC and left insula, contrary to the present study and a previous work (Di Martino et al., 2008). Although this meta-analytic study is of great importance because it first introduced the structure-based meta-analysis technique, the use of different ROIs and statistical methods makes its findings only partially comparable with ours.

There are also notable differences between our study and Di Martino et al.'s (2008) analysis of the rsFC of the striatum. Although most of the findings show considerable overlap, Di Martino et al. described the insulae to be more 
strongly connected with dorsal striatal ROIs and a number of deactivations that we failed to find; interestingly, some of those deactivations are in areas where we found small but significant positive activations, such as the sensorimotor cortex. The IC has been described by Chikama, McFarland, Amaral, and Haber (1997) to have somatotopic anatomic connections with the striatum, wherein the dorsal posterior insula projects to the dorsal putamen whereas the more anterior and ventral insula projects to the caudate nucleus and ventral striatum. These differences are likely to be related to ROIs placed in slightly different positions and multiple regression analyses performed with all the predictors orthogonalized.

The striatum is known to have a ventral-to-dorsal gradient via circuits that spiral from emotional/motivational to decision making and executive motor control (Haber, 2003; Haber et al., 2000). Because of partial volume effects and smoothing, our ROI time courses may be affected by a small but significant residual sampling of more dorsal (i.e., executive/motor) striatal region time courses. However, this bias should also affect MACM, because the results of the former are superimposable to rsFC results. This is remarkable, given the independent nature of these two analyses on fundamentally different types of data as well as the heterogeneity of data contained in BrainMap due to differences in subjects, scanners, analyses, and paradigms. Therefore, the overall convergence of rsFC and MACM results supports the validity of our functional connectivity map.

Interestingly, in our analysis, the right ROI and the left ROI generated a different pattern of connectivity, suggesting that the right NAcc is more connected with uncus, subcallosal gyri, insulae, and parahippocampal gyri, whereas the left NAcc is more connected with OFC, temporal gyri, and PMC. This difference in functional connectivity is consistent with the lateralization pattern emerging from studies with subjects suffering from affective disorders. Converging evidence from neuroimaging, neuropathological and lesion analysis studies revealed predominant involvement of the left MPFC, subgenual ACC, and related limbic and striato-pallido-thalamic structures in regulating emotional expression in cases with recurrent depressive episodes (Drevets, Price, \& Furey, 2008; Drevets, Savitz, \& Trimble, 2008).

Resting state functional connectivity analysis has a number of limitations that merit consideration. First, it has been shown that functional connectivity can change during task performance (Fransson, 2006; De Luca, Smith, De Stefano, Federico, \& Matthews, 2005). Second, resting state analysis faces the same potential limitations as taskrelated fMRI studies with regard to interindividual variability in ventral striatum organization and connectivity. However, the patterns of functional differentiation observed using our seeding approach were reliable and detectable at the individual participant level. Third, our subjects were distributed over a wide age span; however, we attempted to take into account the intersubject varia- bility using a random effect analysis and to reduce the variability induced by age and gender differences by controlling these factors, inserting age and gender as covariates in the statistics. Furthermore, the interpretation of our findings needs caution, because the networks described here are detected in the absence of specific functional activity. We are inferring functional roles for the ventral striatal areas on the basis of their belonging to intrinsic connectivity networks, whose functional relevance is reasonably well established in the literature. Although the exact functional significance of temporal correlations in very low frequency neural fluctuations remains largely unclear, it has been argued that this basal, task-independent, intrinsic connectivity is important to avoid disuse-related pruning of critical synapses (Luo \& O'Leary, 2005) and/or to maintain networks in a primed state, thus improving response efficiency (Fox \& Raichle, 2007). MACM also has limitations. A major limitation of MACM is that this technique identifies regions that tend to be coactivated when NAcc is activated in the absence of a control group. This means that some coactivations might be attributable to common task requirements or mental states beyond reward processing rather than functional connectivity per se.

A recent fMRI study investigated both regional and interregional functional connectivity patterns while subjects performed a gambling task featuring unexpectedly high monetary gains and losses (Camara, Rodriguez-Fornells, $\&$ Munte, 2008). The authors found that monetary gains and losses activated a similar fronto-striato-limbic network, in which main activation peaks were observed bilaterally in the ventral striatum. Our resting state connectivity findings provide confirmation to the role of NAcc as a seat for major "hedonic hotspots" in the widespread pleasure-activated brain networks in humans (Kringelbach \& Berridge, 2009). Such hedonic hotspots are anatomically distributed (NAcc shell and ventral pallidum, other forebrain and limbic cortical regions, and deep brainstem regions including the parabrachial nucleus in the pons) but interact to form a functional integrated circuit, which is sensitive to stimulation with opioids, endocannabinoids, and other neurochemical modulators. From a clinical perspective, the identification of this network is essential to the understanding of the brain mechanisms underlying reward experiences and neuropsychiatric conditions such as addiction, impulse control disorders, and obsessive compulsive spectrum disorders, in addition to disorders of affect (Camara, RodriguezFornells, Ye, \& Münte, 2009). Therefore, our results stress the importance of studying functional connectivity in addition to standard fMRI analysis in reward-related studies in a wide range of neuropsychiatric conditions. Finally, because an assortment of human behaviors is thought to be driven by reward-based processes, including novelty seeking, decision making, economic choice, reinforcement learning, and incentive motivation, future research will be able to show the exact contribution of the NAcc system in the different behavioral contexts to describe both physiological and pathological reward mechanisms more appropriately. 


\section{Methodological Considerations}

Movement was assessed by summing the deviations used to compensate for head motion within the fMRI scanner. The overall quantity of movement was very mild; furthermore, we failed to find a correlation between movement and the age of the subject. Hence, we excluded any influence by these confounds.

In the meta-analysis, a possible confound is generated from the fact that different groups used slightly different templates to transform each subject's MRI or PET image into stereotaxic space. The use of different template brains would result in slight variation in localization of peaks. In particular, depending on the applied normalization procedure, the between-subject variances ranged from 11.0 to $12.1 \mathrm{~mm}$ (Eickhoff et al., 2009), hence the need of a slightly bigger ROIs for the meta-analysis with the respect to the rsFC ROIs.

Our results show good reproducibility: The split half reliability $\left(r_{\mathrm{sb}}=0.73\right)$ and the probabilistic maps (Figure 8) together with the good correspondence observed between the resting state and meta-analytic results lead us to rule out the possibility that the patterns result merely from random fluctuations or that they are due to unintentional tasks by the single subject. In addition, our results are in agreement with anatomical and functional data obtained in previous studies on nonhumans as well as human primates.

\section{Acknowledgments}

We thank all the subjects who participated in this study. The research has been founded by Piedmont Region, Human and Social Science 2008, GIRS project "The invisible grammar of social relations."

Reprint requests should be sent to Franco Cauda, Dipartimento di Psicologia, Via Po 14, 10123 Turin, Italy, or via e-mail: franco. cauda@unito.it.

\section{REFERENCES}

Achard, S., Salvador, R., Whitcher, B., Suckling, J., \& Bullmore, E. (2006). A resilient, low-frequency, small-world human brain functional network with highly connected association cortical hubs. Journal of Neuroscience, 26, 63-72.

Aharon, I., Etcoff, N., Ariely, D., Chabris, C. F., O’Connor, E., \& Breiter, H. C. (2001). Beautiful faces have variable reward value: fMRI and behavioral evidence. Neuron, 32, 537-551.

Alexander, G. E., DeLong, M. R., \& Strick, P. L. (1986). Parallel organization of functionally segregated circuits linking basal ganglia and cortex. Annual Review of Neuroscience, 9, 357-381.

Bandettini, P. A., \& Bullmore, E. (2008). Endogenous oscillations and networks in functional magnetic resonance imaging. Human Brain Mapping, 29, 737-739.

Becerra, L., Breiter, H. C., Wise, R., Gonzalez, R. G., \& Borsook, D. (2001). Reward circuitry activation by noxious thermal stimuli. Neuron, 32, 927-946.

Benjamini, Y., \& Hochberg, Y. (1995). Controlling the false discovery rate: A practical and powerful approach to multiple testing. Royal Statistical Society, Series B, 57, 289-300.

Birn, R. M., Murphy, K., \& Bandettini, P. A. (2008). The effect of respiration variations on independent component analysis results of resting state functional connectivity. Human Brain Mapping, 29, 740-750.

Biswal, B., Yetkin, F. Z., Haughton, V. M., \& Hyde, J. S. (1995). Functional connectivity in the motor cortex of resting human brain using echo-planar MRI. Magnetic Resonance in Medicine, 34, 537-541.

Breiter, H. C., \& Rosen, B. R. (1999). Functional magnetic resonance imaging of brain reward circuitry in the human. Annals of the New York Academy of Sciences, $877,523-547$.

Brog, J. S., Salyapongse, A., Deutch, A. Y., \& Zahm, D. S. (1993). The patterns of afferent innervation of the core and shell in the "accumbens" part of the rat ventral striatum: Immunohistochemical detection of retrogradely transported fluoro-gold. Journal of Comparative Neurology, 338, 255-278.

Brown, W. (1910). Some experimental results in the correlation of mental abilities. British Journal of Psychology, 3, 296-322.

Burgdorf, J., \& Panksepp, J. (2006). The neurobiology of positive emotions. Neuroscience and Biobehavioral Reviews, 30, 173-187.

Camara, E., Rodriguez-Fornells, A., \& Munte, T. F. (2008). Functional connectivity of reward processing in the brain. Frontiers in Human Neuroscience, 2, 19.

Camara, E., Rodriguez-Fornells, A., Ye, Z., \& Münte, T. F. (2009). Reward networks in the brain as captured by connectivity measures. Frontiers in Neuroscience, 3, 350-362.

Cardinal, R. N., Parkinson, J. A., Hall, J., \& Everitt, B. J. (2002). Emotion and motivation: The role of the amygdala, ventral striatum, and prefrontal cortex. Neuroscience and Biobehavioral Reviews, 26, 321-352.

Carlezon, W. A., Jr., \& Thomas, M. J. (2009). Biological substrates of reward and aversion: A nucleus accumbens activity hypothesis. Neuropharmacology, 56(Suppl. 1), 122-132.

Carmichael, S. T., \& Price, J. L. (1994). Architectonic subdivision of the orbital and medial prefrontal cortex in the macaque monkey. Journal of Comparative Neurology, 346, 366-402.

Carretie, L., Albert, J., Lopez-Martin, S., \& Tapia, M. (2009). Negative brain: An integrative review on the neural processes activated by unpleasant stimuli. International Journal of Psychophysiology, 71, 57-63.

Cauda, F., D’Agata, F., Sacco, K., Duca, S., Geminiani, G., \& Vercelli, A. (2011). Functional connectivity of the insula in the resting brain. Neuroimage, 55, 8-23.

Cauda, F., Sacco, K., D’Agata, F., Duca, S., Cocito, D., Geminiani, G., et al. (2009). Low-frequency BOLD fluctuations demonstrate altered thalamocortical connectivity in diabetic neuropathic pain. BMC Neuroscience, 10, 138.

Cavada, C., \& Goldman-Rakic, P. S. (1991). Topographic segregation of corticostriatal projections from posterior parietal subdivisions in the macaque monkey. Neuroscience, 42, 683-696.

Chikama, M., McFarland, N. R., Amaral, D. G., \& Haber, S. N. (1997). Insular cortical projections to functional regions of the striatum correlate with cortical cytoarchitectonic organization in the primate. Journal of Neuroscience, 17, 9686-9705.

Cummings, J. L. (1995). Anatomic and behavioral aspects of frontal-subcortical circuits. Annals of the New York Academy of Sciences, 769, 1-13.

Damoiseaux, J. S., \& Greicius, M. D. (2009). Greater than the sum of its parts: A review of studies combining structural connectivity and resting-state functional connectivity. Brain Structure \& Function, 213, 525-533.

Damoiseaux, J. S., Rombouts, S. A., Barkhof, F., Scheltens, P., Stam, C. J., Smith, S. M., et al. (2006). Consistent resting-state 
networks across healthy subjects. Proceedings of the National Academy of Sciences, U.S.A., 103, 13848-13853.

De Luca, M., Beckmann, C. F., De Stefano, N., Matthews, P. M., \& Smith, S. M. (2006). fMRI resting state networks define distinct modes of long-distance interactions in the human brain. Neuroimage, 29, 1359-1367.

De Luca, M., Smith, S., De Stefano, N., Federico, A., \& Matthews, P. M. (2005). Blood oxygenation level dependent contrast resting state networks are relevant to functional activity in the neocortical sensorimotor system. Experimental Brain Research, 167, 587-594.

de Olmos, J. S., \& Heimer, L. (1999). The concepts of the ventral striatopallidal system and extended amygdala. Annals of the New York Academy of Sciences, 877, 1-32.

Di Martino, A., Scheres, A., Margulies, D. S., Kelly, A. M., Uddin, L. Q., Shehzad, Z., et al. (2008). Functional connectivity of human striatum: A resting state fMRI study. Cerebral Cortex, $18,2735-2747$.

Drevets, W. C., Price, J. L., \& Furey, M. L. (2008). Brain structural and functional abnormalities in mood disorders: Implications for neurocircuitry models of depression. Brain Structure \& Function, 213, 93-118.

Drevets, W. C., Savitz, J., \& Trimble, M. (2008). The subgenual anterior cingulate cortex in mood disorders. CNS Spectrums, 13, 663-681.

Eickhoff, S. B., Laird, A. R., Grefkes, C., Wang, L. E., Zilles, K., \& Fox, P. T. (2009). Coordinate-based activation likelihood estimation meta-analysis of neuroimaging data: A randomeffects approach based on empirical estimates of spatial uncertainty. Human Brain Mapping, 30, 2907-2926.

Ferry, A. T., Ongur, D., An, X., \& Price, J. L. (2000). Prefrontal cortical projections to the striatum in macaque monkeys: Evidence for an organization related to prefrontal networks. Journal of Comparative Neurology, 425, 447-470.

Fox, M. D., \& Raichle, M. E. (2007). Spontaneous fluctuations in brain activity observed with functional magnetic resonance imaging. Nature Reviews Neuroscience, 8, 700-711.

Fox, M. D., Snyder, A. Z., Vincent, J. L., Corbetta, M., Van Essen, D. C., \& Raichle, M. E. (2005). The human brain is intrinsically organized into dynamic, anticorrelated functional networks. Proceedings of the National Academy of Sciences, U.S.A., 102, 9673-9678.

Fox, M. D., Zhang, D., Snyder, A. Z., \& Raichle, M. E. (2009). The global signal and observed anticorrelated resting state brain networks. Journal of Neurophysiology, 101, $3270-3283$.

Fransson, P. (2006). How default is the default mode of brain function? Further evidence from intrinsic BOLD signal fluctuations. Neuropsychologia, 44, 2836-2845.

Friston, K. J., Ashburner, J., Kiebel, S. J., Nichols, T. E., \& Penny, W. D. (Eds.). (2007). Statistical parametric mapping: The analysis of functional brain images. Philadelphia, PA: Academic Press.

Genovese, C. R., Lazar, N. A., \& Nichols, T. (2002). Thresholding of statistical maps in functional neuroimaging using the false discovery rate. Neuroimage, 15, 870-878.

Glimcher, P. W., \& Rustichini, A. (2004). Neuroeconomics: The consilience of brain and decision. Science, 306, 447-452.

Goldman-Rakic, P. S., \& Selemon, L. D. (1986). Topography of corticostriatal projections in nonhuman primates and implications for functional parcellation of the neo-striatum. In E. G. Jones \& A. Peters (Eds.), Cerebral cortex (Vol. 5, pp. 447-466). New York: Plenum Publishing Corp.

Gottfried, J. A., O’Doherty, J., \& Dolan, R. J. (2002). Appetitive and aversive olfactory learning in humans studied using event-related functional magnetic resonance imaging. Journal of Neuroscience, 22, 10829-10837.
Greicius, M. D., Krasnow, B., Reiss, A. L., \& Menon, V. (2003). Functional connectivity in the resting brain: A network analysis of the default mode hypothesis.

Proceedings of the National Academy of Sciences, U.S.A., 100, 253-258.

Greicius, M. D., Supekar, K., Menon, V., \& Dougherty, R. F. (2009). Resting-state functional connectivity reflects structural connectivity in the default mode network. Cerebral Cortex, 19, 72-78.

Grill, J. D., \& Coghill, R. C. (2002). Transient analgesia evoked by noxious stimulus offset. Journal of Neurophysiology, 87, 2205-2208.

Groenewegen, H. J., Wright, C. I., \& Beijer, A. V. (1996). The nucleus accumbens: Gateway for limbic structures to reach the motor system? Progress in Brain Research, 107, 485-511.

Groenewegen, H. J., Wright, C. I., Beijer, A. V., \& Voorn, P. (1999). Convergence and segregation of ventral striatal inputs and outputs. Annals of the New York Academy of Sciences, 877, 49-63.

Haber, S. N. (2003). The primate basal ganglia: Parallel and integrative networks. Journal of Chemical Neuroanatomy, 26, 317-330.

Haber, S. N., Fudge, J. L., \& McFarland, N. R. (2000). Striatonigrostriatal pathways in primates form an ascending spiral from the shell to the dorsolateral striatum. Journal of Neuroscience, 20, 2369-2382.

Haber, S. N., Groenewegen, H. J., Grove, E. A., \& Nauta, W. J. (1985). Efferent connections of the ventral pallidum: Evidence of a dual striato pallidofugal pathway. Journal of Comparative Neurology, 235, 322-335.

Haber, S. N., Kim, K. S., Mailly, P., \& Calzavara, R. (2006). Reward-related cortical inputs define a large striatal region in primates that interface with associative cortical connections, providing a substrate for incentive-based learning. Journal of Neuroscience, 26, 8368-8376.

Haber, S. N., \& McFarland, N. R. (1999). The concept of the ventral striatum in nonhuman primates. Annals of the New York Academy of Sciences, 877, 33-48.

Hagmann, P., Cammoun, L., Gigandet, X., Meuli, R., Honey, C. J., Wedeen, V. J., et al. (2008). Mapping the structural core of human cerebral cortex. PLoS Biology, 6, e159.

Hampson, M., Peterson, B. S., Skudlarski, P., Gatenby, J. C., \& Gore, J. C. (2002). Detection of functional connectivity using temporal correlations in MR images. Human Brain Mapping, 15, 247-262.

Heimer, L., \& Wilson, R. D. (1995). The subcortical projections of the allocortex: Similarities in the neural associations of the hippocampus, the piriform cortex, and the neocortex. In M. Santini (Ed.), Golgi Centennial Symposium: Perspectives in Neurobiology (pp. 177-193). New York: Raven Press.

Heimer, L., Zahm, D. S., \& Alheid, G. F. (1995). Basal ganglia. In G. Paxinos (Ed.), The rat nervous system (2nd ed., pp. 579-628). San Diego; London: Academic Press.

Kienast, T., \& Heinz, A. (2006). Dopamine and the diseased brain. CNS \& Neurological Disorders - Drug Targets, 5, 109-131.

Knutson, B., \& Cooper, J. C. (2005). Functional magnetic resonance imaging of reward prediction. Current Opinion in Neurology, 18, 411-417.

Koob, G. F., Riley, S. J., Smith, S. C., \& Robbins, T. W. (1978). Effects of 6-hydroxydopamine lesions of the nucleus accumbens septi and olfactory tubercle on feeding, locomotor activity, and amphetamine anorexia in the rat. Journal of Comparative \& Physiological Psychology, 92, 917-927.

Koski, L., \& Paus, T. (2000). Functional connectivity of the anterior cingulate cortex within the human frontal lobe: A brain-mapping meta-analysis. Experimental Brain Research, 133, 55-65. 
Kringelbach, M. L., \& Berridge, K. C. (2009). Towards a functional neuroanatomy of pleasure and happiness. Trends in Cognitive Sciences, 13, 479-487.

Laird, A. R., Eickhoff, S. B., Kurth, F., Fox, P. M., Uecker, A. M., Turner, J. A., et al. (2009). ALE meta-analysis workflows via the Brainmap Database: Progress towards a probabilistic functional brain atlas. Frontiers in Neuroinformatics, 3, 23.

Laird, A. R., Eickhoff, S. B., Li, K., Robin, D. A., Glahn, D. C., \& Fox, P. T. (2009). Investigating the functional heterogeneity of the default mode network using coordinate-based meta-analytic modeling. Journal of Neuroscience, 29, 14496-14505.

Laird, A. R., Lancaster, J. L., \& Fox, P. T. (2005). BrainMap: The social evolution of a human brain mapping database. Neuroinformatics, 3, 65-78.

Lancaster, J. L., Laird, A. R., Fox, P. M., Glahn, D. E., \& Fox, P. T. (2005). Automated analysis of meta-analysis networks. Human Brain Mapping, 25, 174-184.

Lancaster, J. L., Woldorff, M. G., Parsons, L. M., Liotti, M., Freitas, C. S., Rainey, L., et al. (2000). Automated Talairach atlas labels for functional brain mapping. Human Brain Mapping, 10, 120-131.

Lawrence, A. D., Sahakianb, B. J., \& Robbins, T. W. (1998). Cognitive functions and corticostriatal circuits: Insights from Huntington's disease. Trends in Cognitive Sciences, 2, 379-388.

Leh, S. E., Ptito, A., Chakravarty, M. M., \& Strafella, A. P. (2007). Fronto-striatal connections in the human brain: A probabilistic diffusion tractography study. Neuroscience Letters, 419, 113-118.

Lehericy, S., Ducros, M., Krainik, A., Francois, C., Van de Moortele, P. F., Ugurbil, K., et al. (2004). 3-D diffusion tensor axonal tracking shows distinct SMA and pre-SMA projections to the human striatum. Cerebral Cortex, 14, 1302-1309.

Luo, L., \& O'Leary, D. D. (2005). Axon retraction and degeneration in development and disease. Annual Review of Neuroscience, 28, 127-156.

Margulies, D. S., Kelly, A. M., Uddin, L. Q., Biswal, B. B., Castellanos, F. X., \& Milham, M. P. (2007). Mapping the functional connectivity of anterior cingulate cortex. Neuroimage, 37, 579-588.

McClure, S. M., York, M. K., \& Montague, P. R. (2004). The neural substrates of reward processing in humans: The modern role of fMRI. Neuroscientist, 10, 260-268.

McFarland, K., Lapish, C. C., \& Kalivas, P. W. (2003). Prefrontal glutamate release into the core of the nucleus accumbens mediates cocaine-induced reinstatement of drug-seeking behavior. Journal of Neuroscience, 23, 3531-3537.

McFarland, N. R., \& Haber, S. N. (2002). Thalamic relay nuclei of the basal ganglia form both reciprocal and nonreciprocal cortical connections, linking multiple frontal cortical areas. Journal of Neuroscience, 22, 8117-8132.

Middleton, F. A., \& Strick, P. L. (1994). Anatomical evidence for cerebellar and basal ganglia involvement in higher cognitive function. Science, 266, 458-461.

Middleton, F. A., \& Strick, P. L. (2002). Basal-ganglia "projections" to the prefrontal cortex of the primate. Cerebral Cortex, 12, 926-935.

Miezin, F. M., Maccotta, L., Ollinger, J. M., Petersen, S. E., \& Buckner, R. L. (2000). Characterizing the hemodynamic response: Effects of presentation rate, sampling procedure, and the possibility of ordering brain activity based on relative timing. Neuroimage, 11, 735-759.

Mobbs, D., Greicius, M. D., Abdel-Azim, E., Menon, V., \& Reiss, A. L. (2003). Humor modulates the mesolimbic reward centers. Neuron, 40, 1041-1048.
Mogenson, G. J., Jones, D. L., \& Yim, C. Y. (1980). From motivation to action: Functional interface between the limbic system and the motor system. Progress in Neurobiology, 14, 69-97.

Morgane, P. J., Galler, J. R., \& Mokler, D. J. (2005). A review of systems and networks of the limbic forebrain/limbic midbrain. Progress in Neurobiology, 75, 143-160.

Napadow, V., Dhond, R., Conti, G., Makris, N., Brown, E. N., \& Barbieri, R. (2008). Brain correlates of autonomic modulation: Combining heart rate variability with fMRI. Neuroimage, 42, 169-177.

O’Donnell, P., \& Grace, A. A. (1995). Synaptic interactions among excitatory afferents to nucleus accumbens neurons: Hippocampal gating of prefrontal cortical input. Journal of Neuroscience, 15, 3622-3639.

O’Donnell, P., Lavin, A., Enquist, L. W., Grace, A. A., \& Card, J. P. (1997). Interconnected parallel circuits between rat nucleus accumbens and thalamus revealed by retrograde transynaptic transport of pseudorabies virus. Journal of Neuroscience, 17, 2143-2167.

Parkinson, J. A., Willoughby, P. J., Robbins, T. W., \& Everitt, B. J. (2000). Disconnection of the anterior cingulate cortex and nucleus accumbens core impairs Pavlovian approach behavior: Further evidence for limbic cortical-ventral striatopallidal systems. Behavioral Neuroscience, 114, 42-63.

Pennartz, C. M., Groenewegen, H. J., \& Lopes da Silva, F. H. (1994). The nucleus accumbens as a complex of functionally distinct neuronal ensembles: An integration of behavioral, electrophysiological and anatomical data. Progress in Neurobiology, 42, 719-761.

Postuma, R. B., \& Dagher, A. (2006). Basal ganglia functional connectivity based on a meta-analysis of 126 positron emission tomography and functional magnetic resonance imaging publications. Cerebral Cortex, 16, 1508-1521.

Redgrave, P., Prescott, T. J., \& Gurney, K. (1999). The basal ganglia: A vertebrate solution to the selection problem? Neuroscience, 89, 1009-1023.

Robbins, T. W., \& Everitt, B. J. (1992). Functions of dopamine in the dorsal and ventral striatum. Seminars in Neuroscience, 4, 119-127.

Robinson, J. L., Laird, A. R., Glahn, D. C., Lovallo, W. R., \& Fox, P. T. (2009). Metaanalytic connectivity modeling: Delineating the functional connectivity of the human amygdala. Human Brain Mapping, 31, 173-184.

Salamone, J. D., Cousins, M. S., \& Snyder, B. J. (1997). Behavioral functions of nucleus accumbens dopamine: Empirical and conceptual problems with the anhedonia hypothesis. Neuroscience and Biobehavioral Reviews, 21, 341-359.

Schultz, W. (2004). Neural coding of basic reward terms of animal learning theory, game theory, microeconomics and behavioral ecology. Current Opinion in Neurobiology, 14, 139-147.

Selemon, L. D., \& Goldman-Rakic, P. S. (1985). Longitudinal topography and interdigitation of corticostriatal projections in the rhesus monkey. Journal of Neuroscience, 5, 776-794.

Smith, S. M., Fox, P. T., Miller, K. L., Glahn, D. C., Fox, P. M., Mackay, C. E., et al. (2009). Correspondence of the brain's functional architecture during activation and rest. Proceedings of the National Academy of Sciences, U.S.A., 106, 13040-13045.

Sorensen, T. (1948). A method of establishing groups of equal amplitude in plant sociology based on similarity of species and its application to analyses of the vegetation on Danish commons. Biologiske Skrifter/Kongelige Danske Videnskabernes Selskab, 5, 1-34.

Spearman, C. (1910). Correlation calculated from faulty data. British Journal of Psychology, 3, 271-295. 
Stoeckel, L. E., Kim, J., Weller, R. E., Cox, J. E., Cook, E. W., III, \& Horwitz, B. (2009). Effective connectivity of a reward network in obese women. Brain Research Bulletin, 79, 388-395.

Surmeier, D. J., Ding, J., Day, M., Wang, Z., \& Shen, W. (2007). D1 and D2 dopamine-receptor modulation of striatal glutamatergic signaling in striatal medium spiny neurons. Trends in Neurosciences, 30, 228-235.

Talairach, J., \& Tournoux, P. (1988). Co-planar stereotaxic atlas of the human brain: 3-Dimensional proportional system: An approach to cerebral imaging. Stuttgart: Thieme.

Taylor, J. R., \& Robbins, T. W. (1984). Enhanced behavioral control by conditioned reinforcers following microinjections of d-amphetamine into the nucleus accumbens. Psychopharmacology (Berlin), 84, 405-412.

Toro, R., Fox, P. T., \& Paus, T. (2008). Functional coactivation map of the human brain. Cerebral Cortex, 18, 2553-2559.

Totterdell, S. (2006). The anatomy of co-morbid neuropsychiatric disorders based on cortico-limbic synaptic interactions. Neurotoxicity Research, 10, 65-85.

Turkeltaub, P. E., Eden, G. F., Jones, K. M., \& Zeffiro, T. A. (2002). Meta-analysis of the functional neuroanatomy of single-word reading: Method and validation. Neuroimage, 16, 765-780.

Vincent, J. L., Patel, G. H., Fox, M. D., Snyder, A. Z., Baker, J. T., Van Essen, D. C., et al. (2007). Intrinsic functional architecture in the anaesthetized monkey brain. Nature, 447, 83-86.

Wise, R. A., Bauco, P., Carlezon, W. A., Jr., \& Trojniar, W. (1992). Self-stimulation and drug reward mechanisms. Annals of the New York Academy of Sciences, 654, 192-198.

Woolrich, M. W., Ripley, B. D., Brady, M., \& Smith, S. M. (2001). Temporal autocorrelation in univariate linear modeling of fMRI data. Neuroimage, 14, 1370-1386.

Zahm, D. S. (1998). Is the caudomedial shell of the nucleus accumbens part of the extended amygdala? A consideration of connections. Critical Reviews in Neurobiology, 12, 245-265.

Zahm, D. S. (1999). Functional-anatomical implications of the nucleus accumbens core and shell subterritories. Annals of the New York Academy of Sciences, 877, 113-128.

Zhang, D., Snyder, A. Z., Fox, M. D., Sansbury, M. W., Shimony, J. S., \& Raichle, M. E. (2008). Intrinsic functional relations between human cerebral cortex and thalamus. Journal of Neurophysiology, 100, 1740-1748. 